\title{
Photoevaporating Proplyd-like objects in Cygnus OB2
}

\author{
Nicholas J. Wright ${ }^{1}$, Jeremy J. Drake ${ }^{1}$, Janet E. Drew ${ }^{2}$, Mario G. Guarcello ${ }^{1}$, \\ Robert A. Gutermuth ${ }^{3}$, Joseph L. Hora ${ }^{1}$, and Kathleen E. Kraemer ${ }^{4}$ \\ 1 Harvard-Smithsonian Center for Astrophysics, 60 Garden Street, Cambridge, MA 02138, \\ USA \\ ${ }^{2}$ Centre for Astronomy Research, Science and Technology Research Institute, University of \\ Hertfordshire, Hatfield AL10 9AB, UK \\ 3 Dept. of Astronomy, University of Massachusetts, Amherst, MA, USA \\ ${ }^{4}$ Boston College, Institute for Scientific Research, 140 Commonwealth Avenue, Chestnut \\ Hill, MA 02467, USA \\ nwright@cfa.harvard.edu
}

\begin{abstract}
We report the discovery of ten proplyd-like objects in the vicinity of the massive OB association Cygnus OB2. They were discovered in IPHAS $\mathrm{H} \alpha$ images and are clearly resolved in broad-band HST/ACS, near-IR and Spitzer mid-IR images. All exhibit the familiar tadpole shape seen in photoevaporating objects such as the Orion proplyds, with a bright ionization front at the head facing the central cluster of massive stars, and a tail stretching in the opposite direction. Many also show secondary ionization fronts, complex tail morphologies or multiple heads. We consider the evidence that these are either proplyds or 'evaporating gaseous globules' (EGGs) left over from a fragmenting molecular cloud, but find that neither scenario fully explains the observations. Typical sizes are 50,000-100,000 AU, larger than the Orion proplyds, but in agreement with the theoretical scaling of proplyd size with distance from the ionizing source. These objects are located at projected separations of $\sim 6-14 \mathrm{pc}$ from the OB association, compared to $\sim 0.1 \mathrm{pc}$ for the Orion proplyds, but are clearly being photoionized by the $\sim 65$ O-type stars in Cyg OB2. Central star candidates are identified in near- and mid-IR images, supporting the proplyd scenario, though their large sizes and notable asymmetries is more consistent with the EGG scenario. A third possibility is therefore considered, that these are a unique class of photoevaporating partially-embedded young stellar objects that have survived the destruction of their natal molecular cloud. This has implications for the properties of stars that form in the vicinity of massive stars.
\end{abstract}


Subject headings: Stars: formation, ISM: jets and outflows, Stars: pre-main sequence, Stars: protostars, Protoplanetary disks, ISM: individual objects: IRAS $20324+4057$

\section{Introduction}

How the star formation process differs in the harsher environments in the Milky Way is of particular interest when considering star formation products such as the initial mass function, the binary fraction, and the fraction of stars that may be home to planetary systems. In recent years evidence has grown that circumstellar disks around young stars are eroded by UV radiation from massive stars (e.g., Johnstone et al. 1998; Guarcello et al. 2007). Since these disks are believed to be the origin of planetary systems such as our own, and the majority of stars that form in our Galaxy do so in regions containing massive stars, this is of particular importance for a global understanding of star formation.

One of the most significant advances in our understanding of the evolution of protoplanetary disks was the discovery of "proplyds" in the Orion Nebula (Laques \& Vidal 1979; Churchwell et al. 1987; O’Dell \& Wen 1994), young stellar objects (YSOs) whose circumstellar material renders them visible either through direct emission or silhouetted against a background His region. The proplyds seen in emission have distinctive tadpole shapes with tails pointing away from $\theta^{1}$ Ori C (O’Dell et al. 1993; O’Dell \& Wong 1996) that are well explained by theoretical models of UV photoevaporation (Johnstone et al. 1998).

Proplyds provide illustrative evidence of the evaporation of circumstellar disks in harsh environments. It is therefore useful to identify them in other systems where photoevaporation might be more widespread. Searches in more massive clusters are hindered by the typically greater distances to them, often leading to the mis-identification of 'evaporating gaseous globules' (EGGs) - fragments of an evaporating molecular cloud - as proplyds (e.g., Smith et al. 2003; De Marco et al. 2006; Smith et al. 2010). Candidate proplyds have so far been identified in NGC 2244, IC 1396, NGC 2264 (all Balog et al. 2006), W5 (Koenig et al. 2008), the Trifid Nebula (Yusef-Zadeh et al. 2005), the Lagoon Nebula (Stecklum et al. 1998), and the Carina star-forming region (Smith et al. 2003), but the best candidates outside of Orion are those in NGC 3603 (Brandner et al. 2000), although these are significantly larger than the Orion proplyds. Many of these regions contain only a single candidate proplyd and never has a true family of proplyds similar to those in the Orion Nebula been found in another region. 
Cygnus OB2 is the most massive young association within $2 \mathrm{kpc}$ of the Sun. It lies within the Cygnus X giant molecular cloud where considerable star formation is ongoing (e.g., Schneider et al. 2006). The association contains >65 O-type stars (Massey \& Thompson 1991; Comerón et al. 2002; Hanson 2003; Kiminki et al. 2007) and therefore provides an excellent sample of the influence of massive stars on the evolution of circumstellar disks. A recent X-ray survey by Wright \& Drake (2009) identified $~ 1500$ lower-mass YSOs, while Wright et al. (2010) found evidence for an age spread among them from 3-5 Myr. This is consistent with evidence for O stars as young as 1 Myr (Hanson 2003), and an older population with an age of 5-7 Myr (Drew et al. 2008). The central cluster has cleared away the majority of molecular gas in the vicinity of the association (Schneider et al. 2006), but recent observations have revealed sites of ongoing star formation around the periphery (e.g., Vink et al. 2008).

In this paper, we present the discovery of 10 objects in the vicinity of Cygnus OB2 that appear remarkably similar to the Orion proplyds. In Section 2 we present the observations used to identify and characterize them, in Section 3 we discuss their physical properties and consider their true nature, and finally in Section 4 we discuss the implications of this finding for our understanding of the propagation of star formation in Cygnus OB2. We adopt a distance of $1.40 \mathrm{kpc}$ for Cyg OB2 (Rygl et al. 2011), whereby $1^{\prime}=0.41 \mathrm{pc}$.

\section{Observations}

The objects presented here were discovered through visual inspection of INT (Isaac Newton Telescope) Photometric H $\alpha$ Survey (IPHAS, Drew et al. 2005) H $\alpha$ images of Cygnus OB2. Here we present the observations that led to their discovery and complementary data at other wavelengths. We enumerate the objects and adopt the IPHAS naming system based on their right ascension and declination. One of these objects, \#5, was identified as an $\mathrm{H} \alpha$ emis-

sion source by Viironen et al. (2009) and labelled IPHASX J203311.5+404141 during an automated search of IPHAS images for compact planetary nebulae.

\subsection{IPHAS $\mathrm{H} \alpha$ Observations}

IPHAS is a survey of the northern Galactic plane in broad-band Sloan $r^{\prime}, i^{\prime}$, and narrowband $\mathrm{H} \alpha$ filters using the INT. Tiling of the $0.25 \mathrm{deg}^{2}$ Wide Field Camera field-of-view leads to almost uninterrupted survey coverage across the Galactic plane at high spatial resolution $\left(1\right.$ pixel $\left.=0.333^{\prime \prime}\right)$. The use of a narrow-band $\mathrm{H} \alpha$ filter $\left(\lambda_{c}=6568 \AA, \mathrm{FWHM}=\right.$ 
$95 \AA$ ) picks out extended emission-line nebulae and point sources (e.g., Wareing et al. 2006; Witham et al. 2008; Sabin et al. 2010). A $1.5 \times 1.5$ degree $\mathrm{H} \alpha$ mosaic of the Cygnus region was produced using MONTAGE, part of which is shown in Figure 1 with the positions of the ten objects discussed in this work indicated. This image was searched for other structures similar to these, but none were found down to the limiting resolution of the image. Individual images of the objects in the $\mathrm{H} \alpha$ filter are shown in Figures 2-4. The $r^{\prime}$ and $i^{\prime}$ broad-band images provide no further information on these diffuse structures and are omitted.

\subsection{UKIDSS near-IR Observations}

Near-IR observations were taken from the UKIRT Infrared Deep Sky Survey (UKIDSS, Lawrence et al. 2007, pixel size 0.4") Galactic Plane Survey (GPS, Lucas et al. 2008). The $J$ and $H$ band images are broadly similar to the deeper $K$-band image shown in Figures $2-4$.

\subsection{Spitzer mid-IR Observations}

The Spitzer Cygnus-X Legacy Survey (Hora et al. 2011) imaged Cygnus X with the Infrared Array Camera (IRAC; Fazio et al. 2004) in four bands (3.6, 4.5, 5.8, and 8.0 $\mu \mathrm{m}$ ) and the Multiband Imaging Photometer for Spitzer (MIPS; Rieke et al. 2004) in the $24 \mu \mathrm{m}$ band. Figures $2-4$ show the objects in the IRAC $8.0 \mu \mathrm{m}$ (pixel size of $1.2^{\prime \prime}$ ), and MIPS $24 \mu \mathrm{m}$ (pixel size of $2.55^{\prime \prime}$ ) bands.

\subsection{HST/ACS Observations}

Object \#7, also known as IRAS 20324+4057, was observed by the Hubble Space Telescope (HST) on 2006 July 22 with the Advanced Camera for Surveys (ACS, 0.05"/ pixel). Observations used the broad-band filters F606W $\left(\lambda_{c}=5907 \AA, \Delta \lambda=2342 \AA\right.$, approximately $V+R)$ and F814W $\left(\lambda_{c}=8333 \AA, \Delta \lambda=2511 \AA\right.$, approximately a broad $I$ filter $)$. Two observations were made in each filter, for a total exposure of $694 \mathrm{~s}$ per filter. A color image of \#7 compiled from these observations is shown in Figure 4 . 


\subsection{Previous identifications}

Some of these objects have previous identifications, either through their extended emission or their central stars, however none have previously been identified as proplyds or EGGs. Some were detected in the infrared, the sub-mm, or radio (see Table 1). Objects 3, 5, and 7 were identified as possible OB stars by Comerón et al. (2002) based on near-IR photometry and low-resolution spectroscopy, suggesting that the central stars of these objects may be more massive than the typical proplyds seen in Orion. They found that \#3 exhibits possible $\mathrm{H}_{2}$ and $\mathrm{CO}$ emission that could originate from a massive circumstellar disk, as the mid-IR images corroborate, while \#7 shows $\mathrm{Br} \gamma$ in emission, most likely originating from the nebulosity. Object \#7 was also imaged by Pereira \& Miranda (2007) during a search for post-AGB candidates and was identified as an His region with a bow shock. Spectra revealed nebular emission lines in a ratio that implied photoionization consistent with ionization by massive stars, rather than shock-excitation.

\section{Discussion}

We now discuss the structure and morphology of the objects and consider the evidence that they are true proplyds like those seen in Orion, or if they are EGGs like those observed by McCaughrean \& Andersen (2002) and De Marco et al. (2006). We note that, unlike many of the Orion proplyds, these objects are all seen in emission and not in silhouette (e.g., McCaughrean \& O'Dell 1996). The silhouettes in Orion are the actual disks themselves, which would not be resolvable at the greater distance of Cygnus OB2 with the observations available. The positions and sizes of these objects are summarized in Table 1 .

\subsection{Morphologies}

All the structures in Figures 2-4 are clearly tadpole shaped with extended tails pointing away from Cyg OB2. They are rim-brightened on the side facing the OB association clearly indicating that they are being photoionized by the massive stars in Cyg OB2. Some show very similar morphologies to Orion proplyds. For example, \#4 shares the 'safety pin' shape of d177-341 (Bally et al. 2000), where the thin sides of the proplyd appear to join up at the end of its tail in a small clump. However, there are minor differences between these proplyds and those in Orion. Objects $2,5, \& 6$ show distinctly non-axisymmetric shapes with many small clumps. The HST image of \#7 in Figure 4 also reveals an interesting non-axisymmetry: the ionization brightness varies between the northern and southern sides 
of its tail. A foreground extinction gradient might cause this, although the required gradient angular size of $\sim 10^{\prime \prime}$ renders this unlikely.

These asymmetries may suggest that these are not proplyds, since the Orion proplyds are clearly symmetric. However, proplyd morphology is determined by the ionizing flux (Johnstone et al. 1998), and here Cygnus OB2 differs significantly from the Orion Nebula or NGC 3603. The massive stars in Cyg OB2 are distributed over a wider area (Figure 1), and not centrally-concentrated like those in other regions, so the ionizing flux emanates from a broader area. Johnstone et al. (1998) showed that the ratio of far-UV to extremeUV flux is important in determining the properties of the resulting photo-evaporative flow and photoionized morphology. Since this ratio varies across the O-type spectral class, the positions of different O-type stars across Cygnus OB2 will result in variation of the flow in different directions and could explain the observed asymmetries.

\subsection{Sizes}

The lengths of these objects range from 18,000 to 113,000 AU (Table 1), making them larger than both the Orion proplyds (40-400 AU, O'Dell 1998), and the hitherto largestknown 'giant proplyds' in NGC 3603 ( 15,000-21,000 AU, Brandner et al. 2000). The absence of smaller objects is a selection effect due to the greater distance of Cyg OB2 and the ground-based imaging from which they were discovered. The large sizes of these objects, orders of magnitude larger than the Orion proplyds, suggests that they could be EGGs, which occupy a large range of sizes.

Despite this the large sizes of these objects can actually be explained by current models of proplyd photoevaporation (Johnstone et al. 1998). Proplyd size depends on how far the ionization front caused by stellar Lyman continuum photons can penetrate into the outflowing photo-dissociation region formed from evaporating circumstellar material (heated by Balmer continuum photons). The size of the proplyd scales inversely with the ionizing extreme UV flux, and therefore should scale with the distance to the source of ionizing radiation, as seen in the Orion Nebula (O’Dell 1998).

While the O stars in Cyg OB2 are spread over a large area, we can approximate the center of the association to be mid-way between the two main groups of massive stars, the northern clump containing Cyg OB2 \#8 and the southern clump dominated by Cyg OB2 \#22 (see e.g., Negueruela et al. 2008). These proplyd-like objects are at projected distances from this center of $\sim 14-34^{\prime}$, or $\sim 6-14$ pc (see Table 1). We find no correlation between this projected distance and the length of the object, suggesting that both these objects and the 
O stars are sufficiently distributed along the line of sight that their projected separations are misleading. However, the typical sizes of the Orion and NGC 3603 proplyds and these objects ( $\sim 100, \sim 20,000$, and $\sim 70,000$ AU respectively) do scale with their projected distances from the source of ionization $(<0.1 \mathrm{pc}, 1-2 \mathrm{pc}$, and $\sim 6-14 \mathrm{pc}$, respectively), supporting a broader correlation.

\subsection{Candidate central stars and the true nature of these objects}

Proplyds, by definition, include an embedded YSO with a protoplanetary disk, while fragments of molecular cloud material may not. In their deep near-IR study of EGGs in the Eagle Nebula, McCaughrean \& Andersen (2002) could only identify protostars in $15 \%$ of the globules, at extinctions up to $A_{V} \sim 27$. This suggests that either most EGGs do not contain central stars, or if they do are so heavily embedded as to escape detection.

To assess the fraction of these objects that contain central stars we searched for point sources in the UKIDSS near-IR images, finding good candidates in 7 of the 10 objects. This fraction is significantly higher than that found by McCaughrean \& Andersen (2002), despite the fact that our observations are $\sim 3$ magnitudes shallower than theirs (the two regions are at similar distances). All these sources are also detected in Spitzer mid-IR observations, suggesting these sources have circumstellar material. The remaining 3 sources appear more complex; \#1 shows mid-IR emission, but is faint and not clearly associated with a central star candidate; \#10 shows only a very faint near-IR point source and no mid-IR source; and \#6 has no good candidate near- or mid-IR source. The detected central source fraction of $70 \%$ appears more consistent with the proplyd scenario than with the EGG scenario.

Many of these point sources are particularly bright at $24 \mu \mathrm{m}$, suggesting a significant mass of cool dust that would be more consistent with embedded Class I sources as opposed to disk-bearing Class II sources. This fact, coupled with the failure of either the proplyd or EGG scenarios to fully explain all the observations, leads us to consider a third scenario. These objects may represent a more advanced stage of EGG evolution, whereby empty EGGs have fully evaporated but those with central stars, which may also be the densest and most massive, have survived the photoevaporation process. This scenario requires further verification but explains many of the observations that the other scenarios do not. If correct it raises new questions such as how the photoevaporation of star-forming EGGs influences the final mass of the embedded stars and how this affects the initial mass function in the vicinity of massive clusters. 


\section{The propagation of star formation in Cygnus}

Regardless of their true nature the presence of clear central star candidates with circumstellar material implies that these objects are very young. If they are proplyds it is well known that irradiated protoplanetary disks are thought to be evaporated in $<1$ Myr (Churchwell et al. 1987; O'Dell et al. 2008), whereas if these are some sort of star-forming EGG they are likely to be even younger. These objects must therefore represent a younger and more-recent population of star formation in the Cygnus region than the slightly older Cyg OB2 association itself. The wide-field view in Figure 1 shows the location of the objects relative to the Cyg OB2 association. The objects are located to the south-east of the main core of the association, approximately half-way between Cyg OB2 and the recently discovered cluster in the HII region DR 15 (Vink et al. 2008), supporting propagation of star formation in a southerly direction. The formation of these objects and the DR 15 cluster may therefore have been triggered by stellar winds or UV radiation from the massive stars in Cyg OB2 or they may have formed anyway and are only now being influenced by Cyg OB2. Differentiating between these two scenarios is important for understand the propagation of star formation in massive clusters and associations such as this.

\section{Summary}

We have presented multi-wavelength images of 10 proplyd-like objects in the vicinity of the nearby massive association Cygnus OB2. Their tadpole-like shapes, clearly visible in IPHAS H $\alpha$ images, are oriented towards the central OB association. We have considered the arguments that these are either true proplyds like those in Orion, or photoionized fragments of molecular cloud material known as EGGs. These objects are notably different from the Orion proplyds, in terms of their larger sizes, asymmetries, and large distances from the source of ionization, but we argue that these factors do not necessarily disprove that they are proplyds and can all be explained under the current model of disk photoevaporation. We show that their large sizes are in agreement with the predicted scaling of proplyd size with distance from the ionizing source, and argue that their asymmetries can be partly explained by the extended distribution of O stars across Cyg OB2 and therefore the complex UV flux distribution. We identify embedded central stars in $70 \%$ of the objects using near- and midIR images, a significantly larger fraction than observed in EGGs in other star forming regions and closer to the $100 \%$ expected for proplyds. We conclude that neither scenario adequately explains the observed properties of these objects and therefore consider a third possibility that these are an intermediate class of object representing the subset of star-forming EGGs that have survived the photoevaporation of the molecular cloud. If this is the case, and these 
objects are still accreting from their natal molecular cloud, it raises the question of whether

photoevaporation from massive stars could influence the initial mass function in regions such as this.

A future paper will present a complete analysis of the photometric properties of the central stars and the long-wavelength emission from circumstellar material, combined with an analysis of the UV radiation field from Cygnus OB2. Further observations may be necessary to determine the true nature of these objects, such as spectroscopy of the central stars and the ionized gas associated with them, or CO observations to ascertain the mass of the gas reservoir.

We thank Geert Barentsen, Robert Greimel, Roberto Raddi, and Jorick Vink for constructive discussions and thank the anonymous referee for a prompt report. This research used data obtained at the INT by the IPHAS project and processed by the Cambridge Astronomical Survey Unit. We acknowledge data products from the Spitzer Space Telescope, UKIDSS, and the NASA/ESA Hubble Space Telescope, and MONTAGE, funded by NASA. JJD was supported by NASA contract NAS8-39073 to the Chandra X-ray Center (CXC).

\section{REFERENCES}

Bally, J., O’Dell, C. R., \& McCaughrean, M. J. 2000, AJ, 119, 2919

Balog, Z., Rieke, G. H., Su, K. Y. L., Muzerolle, J., \& Young, E. T. 2006, ApJ, 650, L83

Brandner, W., Grebel, E. K., Chu, Y.-H., et al. 2000, AJ, 119, 292

Churchwell, E., Felli, M., Wood, D. O. S., \& Massi, M. 1987, ApJ, 321, 516

Comerón, F., Pasquali, A., Rodighiero, G., et al. 2002, A\&A, 389, 874

De Marco, O., O’Dell, C. R., Gelfond, P., Rubin, R. H., \& Glover, S. C. O. 2006, AJ, 131, 2580

Drew, J. E., Greimel, R., Irwin, M. J., et al. 2005, MNRAS, 362, 753

Drew, J. E., Greimel, R., Irwin, M. J., \& Sale, S. E. 2008, MNRAS, 386, 1761

Fazio, G. G., Hora, J. L., Allen, L. E., et al. 2004, ApJS, 154, 10

Guarcello, M. G., Prisinzano, L., Micela, G., et al. 2007, A\&A, 462, 245 
Hanson, M. M. 2003, ApJ, 597, 957

Hora, J. L., Gutermuth, R. A., Carey, S., et al. 2011, in Reionization to Exoplanets, ASP Conf. Series, in press.

Johnstone, D., Hollenbach, D., \& Bally, J. 1998, ApJ, 499, 758

Kiminki, D. C., Kobulnicky, H. A., Kinemuchi, K., et al. 2007, ApJ, 664, 1102

Koenig, X. P., Allen, L. E., Kenyon, S. J., Su, K. Y. L., Balog, Z. 2008, ApJ, 687, L37

Laques, P., \& Vidal, J. L. 1979, A\&A, 73, 97

Lawrence, A., Warren, S. J., Almaini, O., et al. 2007, MNRAS, 379, 1599

Lucas, P. W., Hoare, M. G., Longmore, A., et al. 2008, MNRAS, 391, 136

Massey, P., \& Thompson, A. B. 1991, AJ, 101, 1408

McCaughrean, M. J., \& Andersen, M., A\&A, 389, 513

McCaughrean, M. J., \& O’Dell, C. R. 1996, AJ, 111, 1977

Negueruela, I., Marco, A., Herrero, A., \& Clark, J. S. 2008, A\&A, 487, 575

O’Dell, C. R. 1998, AJ, 115, 263

O’Dell, C. R., Muench, A., Smith, N., \& Zapata, L. 2008, in Handbook of Star Forming Regions, Volume I, ed. Reipurth, B. (Astronomical Society of the Pacific), 544

O’dell, C. R., \& Wen, Z. 1994, ApJ, 436, 194

O’Dell, C. R., Wen, Z., \& Hu, X. 1993, ApJ, 410, 696

O’Dell, C. R., \& Wong, K. 1996, AJ, 111, 846

Pereira, C. B., \& Miranda, L. F. 2007, A\&A, 462, 231

Rieke, G. H., Young, E. T., Engelbracht, C. W., et al. 2004, ApJS, 154, 25

Rosolowsky, E., Dunham, M. K., Ginsburg, A., et al. 2010, ApJS, 188, 123

Rygl, K. L. J., Brunthaler, A., Sanna, A., et al. 2011, arXiv e-prints 1111.7023.

Sabin, L., Zijlstra, A. A., Wareing, C., et al. 2010, PASA, 27, 166

Schneider, N., Bontemps, S., Simon, R., et al. 2006, A\&A, 458, 855 
Smith, N., Bally, J., \& Morse, J. A. 2003, ApJ, 587, L105

Smith, N., Povich, M. S., Whitney, B. A., et al. 2010, MNRAS, 406, 952

Stecklum, B., Henning, T., Feldt, M., et al. 1998, AJ, 115, 767

Viironen, K., Greimel, R., Corradi, R. L. M., et al. 2009, A\&A, 504, 291

Vink, J. S., Drew, J. E., Steeghs, D., et al. 2008, MNRAS, 387, 308

Wareing, C. J., O'Brien, T. J., Zijlstra, A. A., et al. 2006, MNRAS, 366, 387

Wendker, H. J., Higgs, L. A., \& Landecker, T. L. 1991, A\&A, 241, 551

Witham, A. R., Knigge, C., Drew, J. E., et al. 2008, MNRAS, 384, 1277

Wright, N. J., \& Drake, J. J. 2009, ApJS, 184, 84

Wright, N. J., Drake, J. J., Drew, J. E., \& Vink, J. S. 2010, ApJ, 713, 871

Yusef-Zadeh, F., Biretta, J., \& Geballe, T. R. 2005, AJ, 130, 1171 


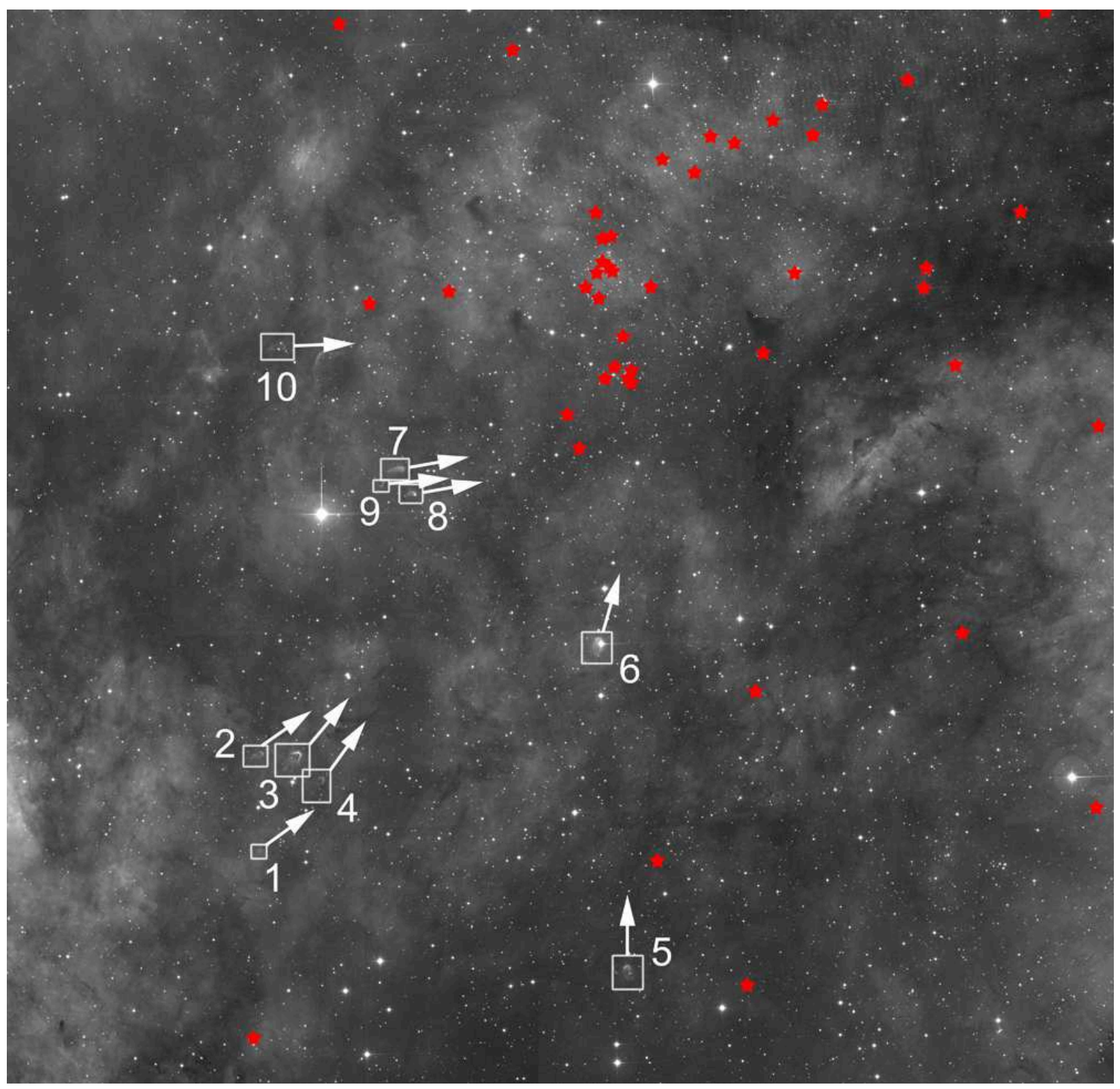

Fig. 1.- IPHAS H $\alpha$ image of the Cygnus OB2 region displayed using a logarithmic intensity scale. The image is $1^{\circ} \times 1^{\circ}(\sim 24 \times 24 \mathrm{pc}$ at $1.40 \mathrm{kpc})$ and centered on (R.A., Dec) $=$ (20:33:20, +41:05:00) with north up and east to the left. The locations of the ten objects are indicated and numbered, with arrows showing the direction of their semi-major axes and ionization fronts. The positions of all known O-type stars are shown as red stars. DR 15 is approximately $20^{\prime}$ south of this image. 

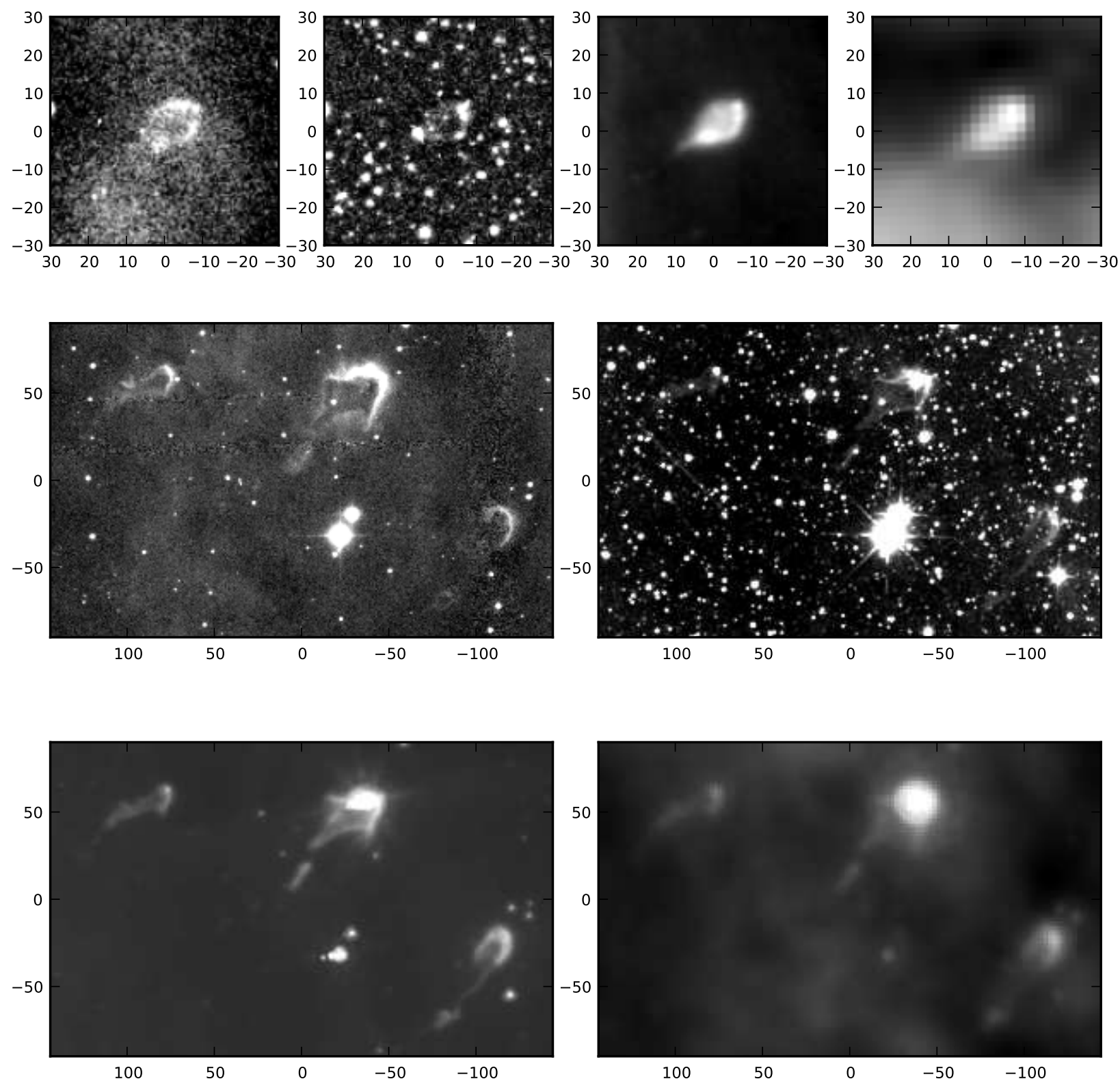

Fig. 2. - Images of objects \#1-4 at different wavelengths. Top: Object \#1, from left to right: IPHAS H $\alpha$, UKIDSS K-band, IRAC $8 \mu \mathrm{m}$, MIPS $24 \mu \mathrm{m}$. Middle: Objects \#2-4 in IPHAS $\mathrm{H} \alpha$ (left) and UKIDSS K-band (right). Bottom: Objects \#2-4 in IRAC $8 \mu \mathrm{m}$ (left) and MIPS $24 \mu \mathrm{m}$. All images are shown with north up and east to the left and with axes in arcseconds offset from the center of each image. At a distance of $1.40 \mathrm{kpc}$, $10^{\prime \prime} \sim 1.4 \times 10^{4} \mathrm{AU} \sim 0.07 \mathrm{pc}$. 

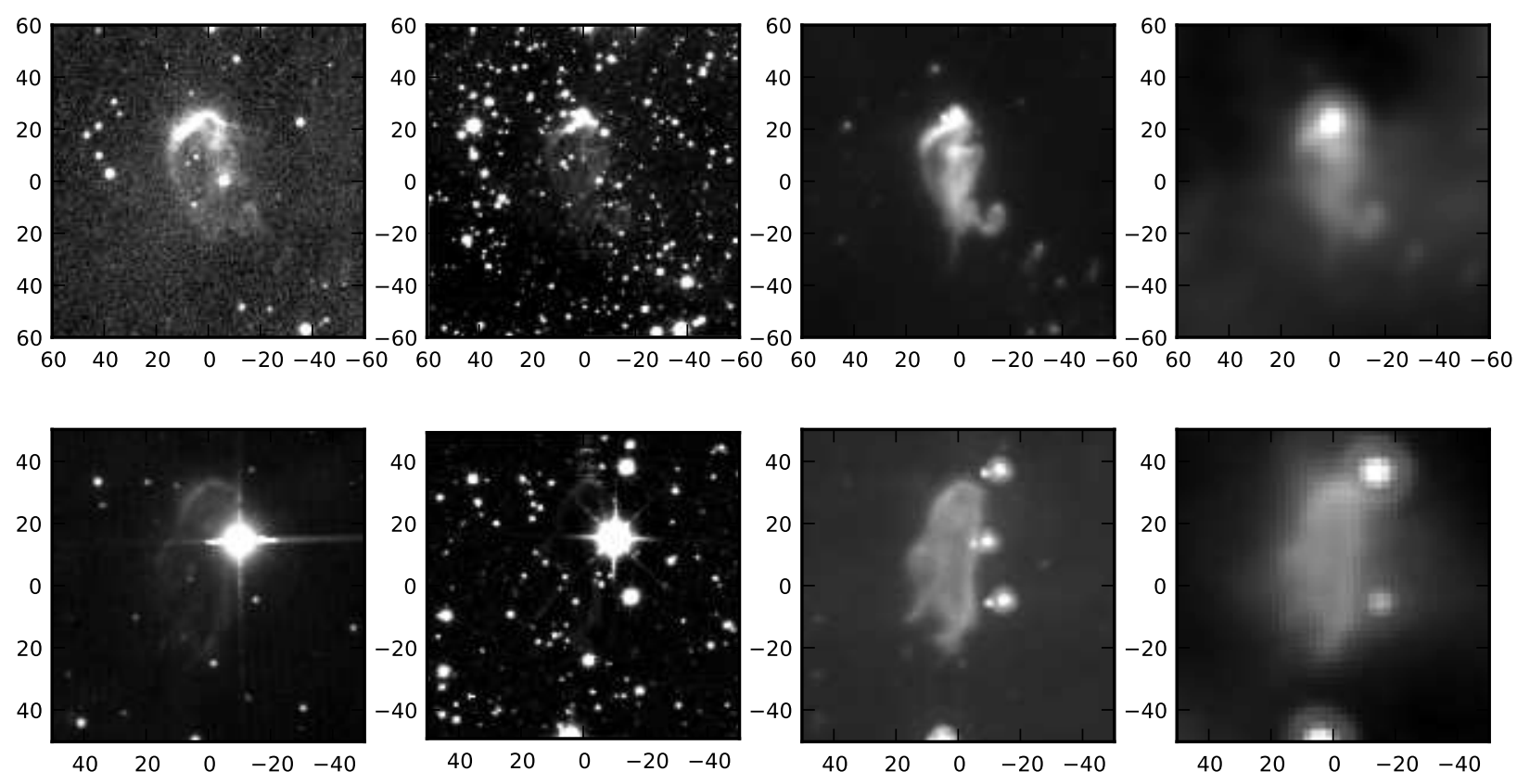

Fig. 3.- Images of objects \#5 (top) and \#6 (bottom) at different wavelengths, from left to right: IPHAS $\mathrm{H} \alpha$, UKIDSS K-band, IRAC $8 \mu \mathrm{m}$, MIPS $24 \mu \mathrm{m}$. All images are shown with north up and east to the left and with axes in arcseconds offset from the center of each image. At a distance of $1.40 \mathrm{kpc}, 10^{\prime \prime} \sim 1.4 \times 10^{4} \mathrm{AU} \sim 0.07 \mathrm{pc}$. 

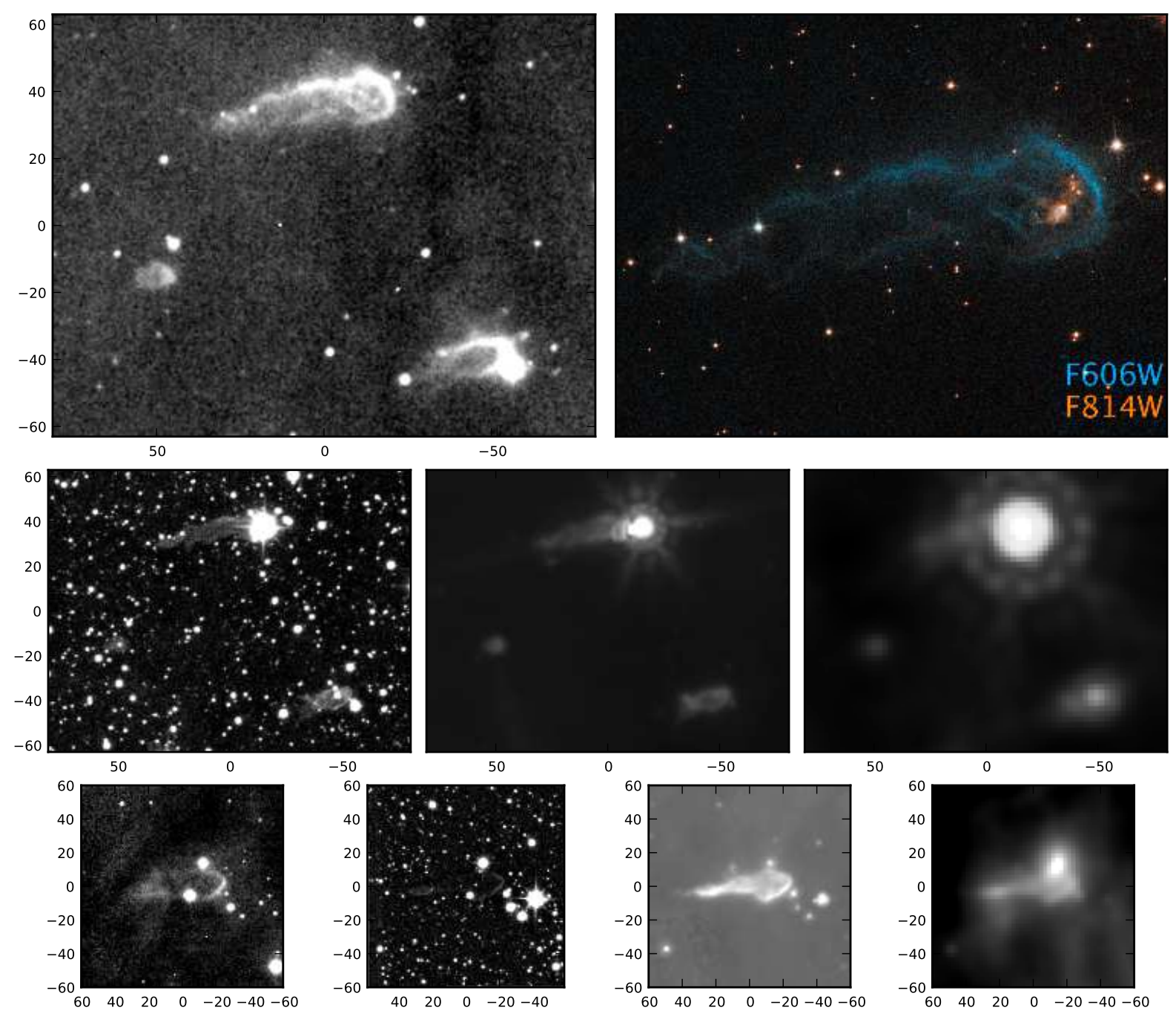

Fig. 4.- Images of objects \#7-10 at different wavelengths. Top left: Objects \#7-9 in IPHAS H $\alpha$. Top right: HST/ACS color image of object \#7 (courtesy Z. Levay and L. Frattare, STScI) at a pixel scale of $0.05^{\prime \prime}\left(65^{\prime \prime} \times 50^{\prime \prime} \sim 0.44 \times 0.34 \mathrm{pc}\right)$. Middle: Objects \#7-9 in (from left to right) UKIDSS K-band, IRAC $8 \mu \mathrm{m}$, and MIPS $24 \mu \mathrm{m}$. Bottom: Object \#10, from left to right: IPHAS H $\alpha$, UKIDSS K-band, IRAC $8 \mu \mathrm{m}$, MIPS $24 \mu \mathrm{m}$. All images are shown with north up and east to the left and with axes in arcseconds offset from the centers. At a distance of $1.40 \mathrm{kpc}, 10^{\prime \prime} \sim 1.4 \times 10^{4} \mathrm{AU} \sim 0.07 \mathrm{pc}$. 
Table 1: Properties of the objects identified in the vicinity of Cygnus OB2.

\begin{tabular}{llccccc}
\hline$\#$ & Name & $\begin{array}{c}\text { P.A. } \\
\left({ }^{\circ}\right)\end{array}$ & $\begin{array}{c}\text { Length } \\
\left(10^{3} \mathrm{AU}\right)\end{array}$ & $\begin{array}{c}\text { Width } \\
\left(10^{3} \mathrm{AU}\right)\end{array}$ & $\begin{array}{c}\text { Projected } \\
\text { distance }(\mathrm{pc})\end{array}$ & Notes \\
\hline 1 & IPHASX J203453.6+404814 & 54 & 24 & 16 & 13.7 & \\
2 & IPHASX J203453.3+405321 & 55 & 63 & 22 & 12.0 & \\
3 & IPHASX J203443.2+405313 & 40 & 113 & 60 & 11.6 & IRAS 20328+4042. Radio source (Wendker et al. 1991). \\
4 & IPHASX J203436.4+405154 & 36 & 102 & 32 & 11.7 & \\
5 & IPHASX J203311.5+404141 & 0 & 75 & 41 & 13.9 & \\
6 & IPHASX J203318.8+405905 & 16 & 77 & 27 & 6.9 & Sub-mm source (Rosolowskv et al. 2010). \\
7 & IPHASX J203413.3+410814 & 80 & 77 & 22 & 5.6 & IRAS 20324+4057. \\
8 & IPHASX J203410.5+410659 & 80 & 43 & 15 & 5.8 & \\
9 & IPHASX J203419.0+410722 & 85 & 18 & 13 & 6.2 & \\
10 & IPHASX J203447.4+411445 & 88 & 83 & 27 & 7.2 & IRAS 20329+4104. \\
\hline
\end{tabular}

The source name is provided in the IPHAS sexagesimal, equatorial position-based format for extended objects: IPHASXJhhmmss.s+ddmmss, with "J" indicating the position is J2000. The position angle (P.A.) is given as the angle measured clockwise between North and the apparent major-axis of the object. The dimensions of the objects and the projected distance to the center of Cyg OB2 have been calculated using a distance of $1.40 \mathrm{kpc}$ (Hanson 2003). 